\title{
Silkworm model for Bacillus anthracis infection and virulence determination
}

Atmika Paudel*, Yoshikazu Furuta, Hideaki Higashi

Division of Infection and Immunity, International Institute for Zoonosis Control, Hokkaido University, Sapporo, Hokkaido, Japan

*Address correspondence to: Atmika Paudel, atmikapd@czc.hokudai.ac.jp

North 20, West 10, Kita-ku, Sapporo, 001-0020, Hokkaido, Japan

Tel: 011-706-9497, FAX: 011-706-9548

Keywords: Bacillus anthracis, Bombyx mori, silkworm, virulence, animal model, hostpathogen interaction. 


\section{Abstract}

Bacillus anthracis is an obligate pathogen and a causative agent of anthrax. Its major virulence factors are plasmid-coded; however, recent studies have revealed chromosome-encoded virulence factors, indicating that the current understanding of its virulence mechanism is elusive and needs further investigation. In this study, we established a silkworm (Bombyx mori) infection model of $B$. anthracis Sterne. We showed that silkworms were killed by $B$. anthracis and cured of the infection when administered with antibiotics. We quantitatively determined the lethal dose of the bacteria that kills $50 \%$ larvae and effective doses of antibiotics that cure $50 \%$ infected larvae. Furthermore, we demonstrated that $B$. anthracis mutants with disruption in virulence genes such as pagA, lef, and atxA had attenuated silkworm-killing ability and reduced colonization in silkworm hemolymph. The silkworm infection model established in this study can be utilized in large-scale infection experiments to identify novel virulence determinants and develop novel therapeutic options against $B$. anthracis infections.

\section{Introduction}

Bacillus anthracis is a spore-forming Gram-positive bacterium that infects both animals and humans. Most animals ingest $B$. anthracis spores while grazing and develop an infection, and humans occasionally acquire infection from the infected animals or animal products. The spore-forming ability allows the bacteria to exist for decades in a dormant state and resist harsh environments ${ }^{1}$. Despite immunization efforts, $B$. anthracis is still a potential threat due to sporadic anthrax outbreaks ${ }^{2-9}$ and its use in bioterrorism ${ }^{10}$. Consequently, the world health organization and centers for disease control and prevention have placed $B$. anthracis as one of the top bioterrorism agents.

The pathogenicity of $B$. anthracis has been attributed mainly to toxins and capsule encoded in the plasmids $\mathrm{pXO} 1$ and $\mathrm{pXO}$, respectively ${ }^{11,12}$. Emerging evidence has uncovered the involvement of chromosomal genes in virulence ${ }^{13-18}$, implying that other virulence factors of $B$. anthracis are yet to be identified. As pathogenesis is an outcome of host-pathogen interaction, a suitable animal host is desired to understand the 
virulence mechanism and design novel therapeutic approaches. A model that can be used in large numbers and have fewer ethical concerns would be of particular importance during the initial phases of research. Due to economic, technical, and ethical concerns associated with the use of vertebrate animals, scientists are turning toward invertebrate animal models such as Caenorhabditis elegans ${ }^{19-21}$, Drosophila melanogaster ${ }^{22-24}$, Galleria mellonella ${ }^{25-27}$ and Bombyx mori28-30 for large scale screenings. While accurate dose administration in $C$. elegans and $D$. melanogaster is difficult due to their small size, the use of $G$. mellonella is challenging due to its faster locomotion. Bombyx mori (silkworm) larvae have been used as a desirable animal model with many physical and biological advantages ${ }^{31}$. They are small enough for easy handling yet large to perform experiments involving organ isolations and desired quantity injections. Besides, due to their slow locomotion and harmless nature, they have less biohazard potential. Conserved basic biological features with mammals make silkworms appropriate as animal models of human diseases ${ }^{32-34}$. Using silkworms as an infection model, novel antimicrobial agents and novel genes with roles in bacterial virulence have been identified ${ }^{28,29,35-38}$.

In this study, we established a silkworm model of $B$. anthracis infection. We demonstrated that $B$. anthracis kills silkworms, and the infection can be treated by clinically used antibiotics. Using fluorescence protein-expressing strain, we revealed how $B$. anthracis establishes infection inside the host. We further showed that mutants with disruption in the genes encoding known virulence factors had decreased virulence in silkworms.

\section{Materials and Methods}

\section{Bacterial strains and culture condition}

The bacterial strains used in this study are shown in Table 1. B. anthracis 34F2 with pRP1099, a plasmid possessing the gene for AmCyan1 protein, was constructed by conjugation $^{39}$. Strains were grown in Brain-Heart Infusion (BHI) medium (Difco, USA) for 
routine culture at $37^{\circ} \mathrm{C}$. Kanamycin $(20 \mu \mathrm{g} / \mathrm{ml})$ was supplemented for BYF10124. For liquid cultures, strains were grown at $37^{\circ} \mathrm{C}$ with shaking at $155 \mathrm{rpm}$.

Table 1: Bacterial strains used in this study

\begin{tabular}{|c|c|c|}
\hline Strain & Description & Reference \\
\hline 34F2 & Bacillus anthracis Sterne, vaccine strain $\left(\mathrm{pXO} 1^{+}, \mathrm{pXO2}\right)$ & Sterne et al. ${ }^{40}$ \\
\hline BYF10008 & Strain derived from $34 \mathrm{~F} 2, \Delta$ pagA & Furuta et al. ${ }^{41}$ \\
\hline BYF10054 & Strain derived from $34 \mathrm{~F} 2, \triangle a t x A$ & Furuta et $a l^{41}$ \\
\hline BYF10009 & Strain derived from $34 \mathrm{~F} 2, \Delta / e f$ & 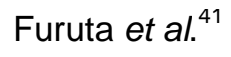 \\
\hline BYF10124 & $\begin{array}{l}\text { 34F2 harboring pRP1099, expressing fluorescence } \\
\text { protein AmCyan1 }\end{array}$ & This study \\
\hline
\end{tabular}

\section{Silkworm rearing}

Silkworm eggs were purchased from Ehime-Sanshu Co., Ltd. (Ehime, Japan), disinfected, and reared at $27^{\circ} \mathrm{C}$. The worms were fed with antibiotic-containing artificial diet Silkmate 2S (Nihon Nosan Corp., Japan) until the fifth instar stage as previously described $^{36}$. After the larvae turn to the fifth instar, they were fed an antibiotic-free artificial diet (Sysmex, Japan) and used for infection experiments the following day.

\section{Silkworm infection experiments}

For infection experiments, fifth-instar day-2 silkworm larvae were used. Bacterial strains were revived from glycerol stock by streaking them on $\mathrm{BHI}$ agar plates and incubating overnight at $37^{\circ} \mathrm{C}$. The overnight grown single colony was inoculated and cultured overnight in $5 \mathrm{ml} \mathrm{BHI}$ medium with shaking at $155 \mathrm{rpm}$. The culture was 100-fold diluted in $\mathrm{BHI}$ medium and grown till the $\mathrm{OD}_{600}$ reached 0.5 . The cells were diluted with physiological saline solution $(0.9 \% \mathrm{NaCl})$, where applicable, and $50 \mu \mathrm{l}$ of bacterial suspension was injected into the hemolymph of each larva using a 1-ml syringe equipped with a 27-gauze needle (Terumo Medical Corporation, Japan). Silkworms were considered dead if they did not move when poked with forceps.

\section{Antimicrobial susceptibility test}

Antibiotics were obtained from either Fujifilm Wako, Japan, or Sigma Aldrich, Japan. Antimicrobial susceptibility test was performed by broth micro-dilution assay according 
to the Clinical and Laboratory Standards Institute protocol (CLSI) as explained previously ${ }^{36}$. The plate was incubated at $37^{\circ} \mathrm{C}$ for $20 \mathrm{~h}$, and the minimum inhibitory concentration (MIC) of each antibiotic was determined as the minimum concentration that inhibited the growth of bacteria.

\section{Treatment of infection by antibiotics in silkworms}

To evaluate the therapeutic activities of clinically used antibiotics in the infected silkworms, exponentially growing bacteria $\left(\sim 5 \times 10^{2} \mathrm{CFU}\right)$ was injected into the hemolymph of each larva. Different concentration of antibiotics was prepared in saline and injected to the larvae into the hemolymph within $30 \mathrm{~min}$ of infection. For survival assay, $1 \mathrm{mg} / \mathrm{kg}$ of doxycycline and ampicillin each were injected into the larvae. To determine effective doses that cure $50 \%$ larvae $\left(E D_{50}\right)$, various concentrations of doxycycline and ampicillin were prepared in saline and injected into the hemolymph of infected larvae ( $n=3$ for each dose). The survival of larvae was recorded, and $E D_{50}$ was calculated from the survival at $16 \mathrm{~h}$ post-infection by logistic regression analysis using the logit link function. To determine the microbial burden, larvae infected with $B$.

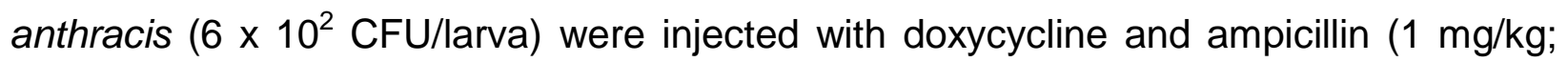
$\mathrm{n}=10$ ) within 30 min of infection, hemolymph was collected $6 \mathrm{~h}$ and $9 \mathrm{~h}$ post-injection, diluted with saline and spread on Luria-Bertani agar plates followed by overnight incubation at $37^{\circ} \mathrm{C}$. The appearing colonies were counted the next day.

\section{Fluorescence imaging}

B. anthracis BYF10124 was injected into silkworm hemolymph. The silkworms were kept at $27^{\circ} \mathrm{C}$. After $3 \mathrm{~h}$ and $6 \mathrm{~h}$ post-infection, hemolymph from the infected silkworm was collected, placed on a glass slide, and covered by a coverslip. Fluorescence images of the samples were collected using an inverted Zeiss LSM 780 confocal microscope equipped with an EM-CDD camera (Zeiss Research Microscopy Solutions, Germany) under a 40 x objective lens. To determine the effect of the antibiotic, $1 \mathrm{mg} / \mathrm{kg}$ ampicillin was injected into the infected larvae, and hemolymph was observed under the microscope. 


\section{Assessment of virulence in silkworms}

Virulence of the bacterial strains in silkworms was tested by injecting the exponentially growing $B$. anthracis 34F2 and mutants with disruption in virulence genes $\left(\sim 5 \times 10^{2}\right.$ $\mathrm{CFU}$ ) into the hemolymph of each larva. For survival, larvae were observed at different time intervals post-infection. To determine microbial burden, hemolymph of the infected larva was collected at $3 \mathrm{~h}$ and $6 \mathrm{~h}$ post-infection, diluted in saline, and colony-forming units were determined.

\section{Results}

\section{Silkworms are killed by Bacillus anthracis infection}

To establish a silkworm infection model of $B$. anthracis, we injected silkworm larvae with different cell numbers of $B$. anthracis Sterne strain 34F2 into the hemolymph and checked the survival. We found that $B$. anthracis killed the silkworms in a dosedependent manner (Fig. 1a). We determined the lethal dose that killed $50 \%$ of the worms $\left(\mathrm{LD}_{50}\right)$ 16h post-infection to be $8.3 \times 10^{2}$ colony forming units (CFU) per larva (Fig. 1b). At 19h post-infection, when all the silkworms infected with $8.1 \times 10^{2} \mathrm{CFU}$ of $B$. anthracis died (Fig. 1c), saline-injected silkworms were still surviving (Fig. 1d), indicating that the fatality is brought about by $B$. anthracis infection. The death of larvae was accompanied by a change of skin color to black due to melanization. 
a.

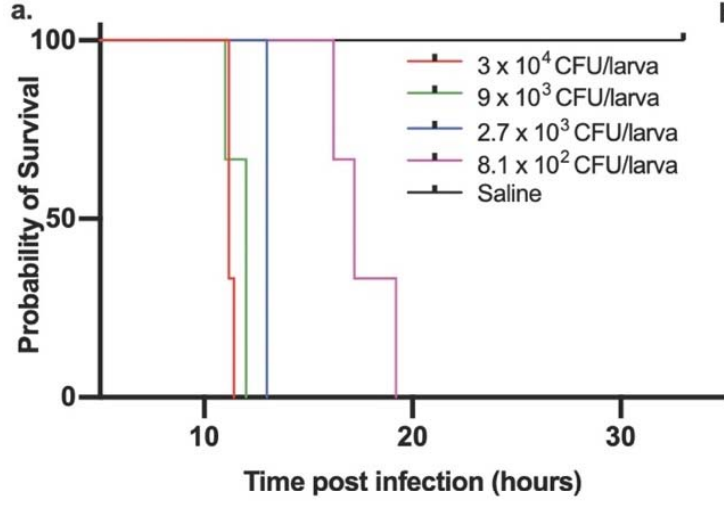

c.

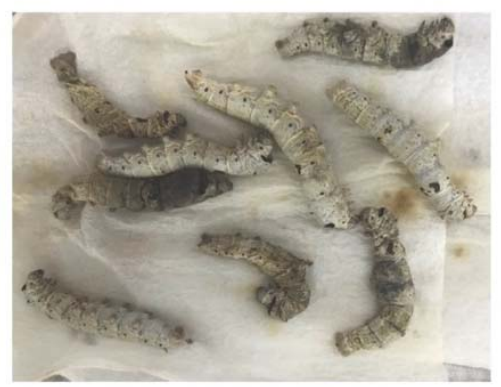

B. anthracis infected b.

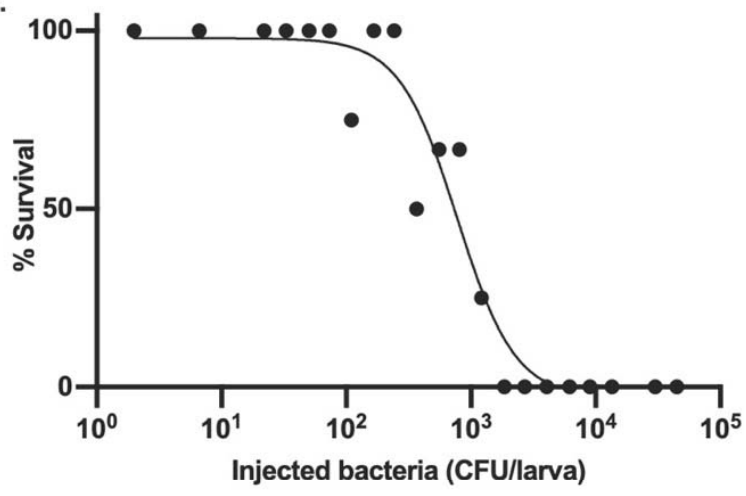

d.

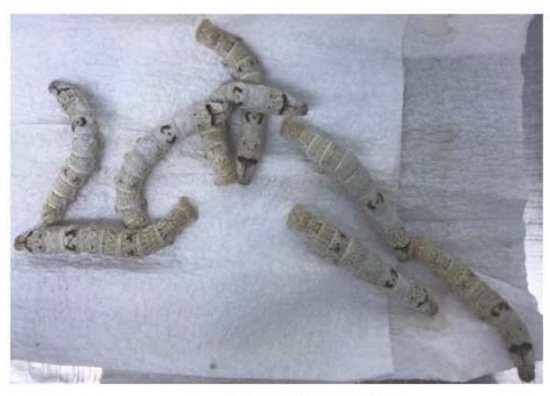

Saline injected

Figure 1: Bacillus anthracis kills silkworms. a. Dose-dependent killing of silkworms by $B$. anthracis. Data is representative of three independent experiments. b. Survival of silkworms $16 \mathrm{~h}$ post-infection. Data is presented as a combined result of three independent experiments. $L_{50}$ is calculated by logistic regression analysis using the logit link function. c. Dead silkworms infected with $B$. anthracis $\left(8.1 \times 10^{2}\right.$ CFU/larva) $19 \mathrm{~h}$ post-infection. The dead silkworms turn black due to melanization. d. Alive silkworms injected with saline $19 \mathrm{~h}$ post-injection.

To further confirm that the observed killing of silkworms was due to $B$. anthracis infection, we heat-killed the bacteria by autoclaving and injected into the silkworms. We found that injection of heat-killed bacteria equivalent to $1.5 \times 10^{6} \mathrm{CFU}$ did not kill the worms while live $2.6 \times 10^{3} \mathrm{CFU}$ killed the worms within $16 \mathrm{~h}$ post-infection (Fig. 2a). We further constructed fluorescence protein AmCyan1-expressing B. anthracis, whose silkworm killing ability was similar to that of the wild-type (Fig. 2b). We confirmed the florescence-protein expression by observing the cells under a fluorescence microscope (Fig. 2c). We then infected the silkworm with the bacteria, recovered hemolymph $3 \mathrm{~h}$ and $6 \mathrm{~h}$ post-infection, and confirmed the fluorescence expression of bacteria in the hemolymph. While most of the bacteria were engulfed by hemocytes $3 \mathrm{~h}$ post-infection (Fig. 2d), increased bacterial growth outside the hemocytes was observed 6h post- 
infection (Fig. 2e), indicating the progression of bacterial proliferation within the host and establishment of infection by the bacteria.

a.

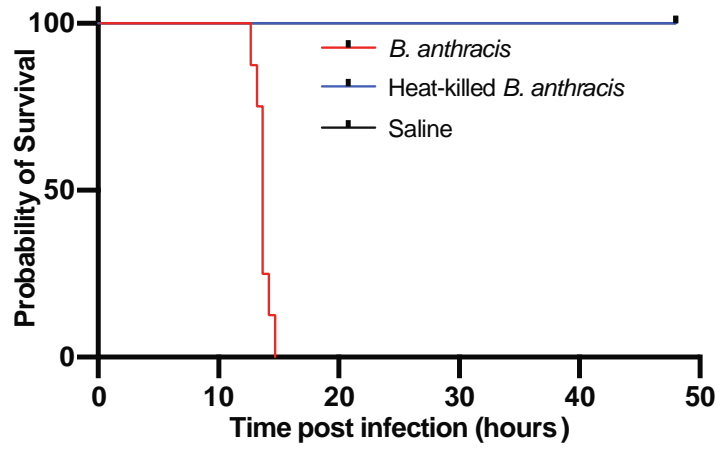

b.

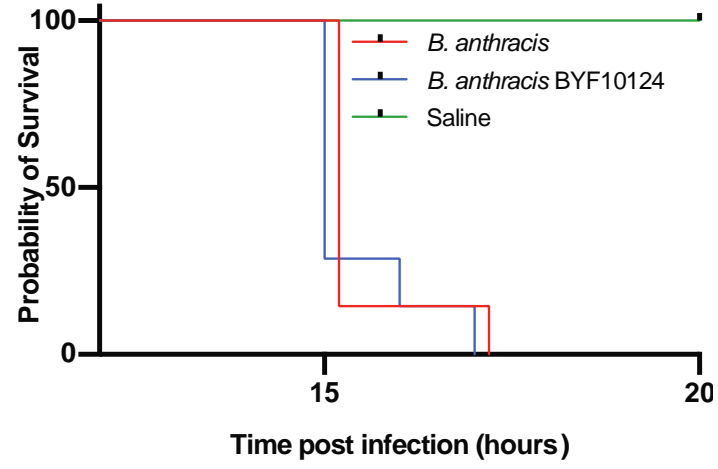

c.

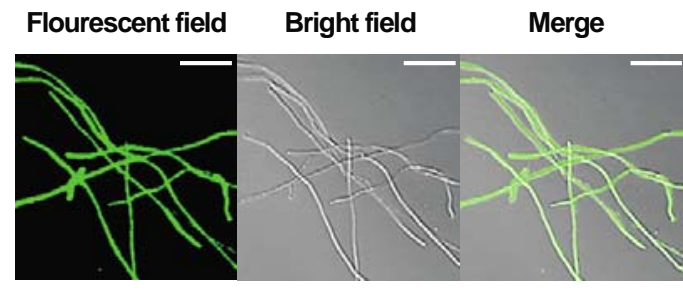

d.

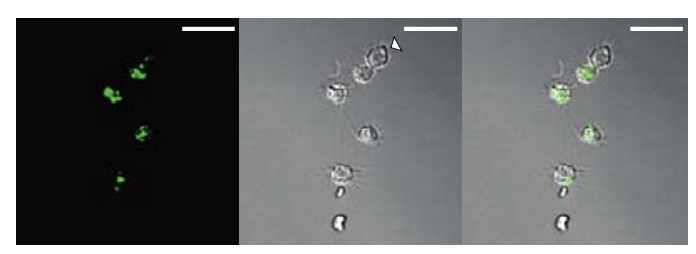

e.
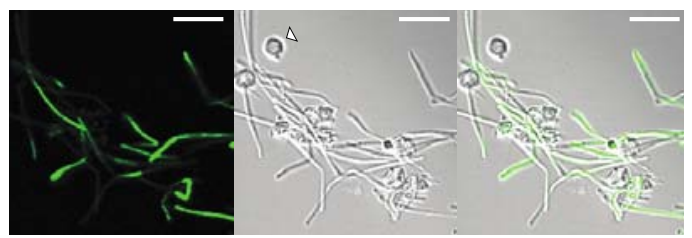

Figure 2: Live bacteria is required for silkworm killing. a. Survival of silkworms after injection of heatkilled B. anthracis. Live B. anthracis $\left(2.6 \times 10^{3}\right.$ CFU/larva) and heat-killed B. anthracis $\left(1.5 \times 10^{6}\right.$ CFU/larva) were injected to silkworms $(n=8)$, and survival was observed. Representative data of two independent experiments are shown. b. Survival of silkworms after infection with wild-type and BYF10124. Wild-type $B$. anthracis $\left(5 \times 10^{2} \mathrm{CFU} /\right.$ larva) and BYF10124 (8.5 × $10^{2} \mathrm{CFU} /$ larva) were injected to silkworms $(n=7)$, and survival was observed. Representative data of two independent experiments are shown c. In vitro grown BYF10124 under microscope. d, e. BYF10124 in silkworm hemolymph $3 \mathrm{~h}$ (d) and 6h (e) post-infection under microscope. White arrows indicate hemocytes of silkworm hemolymph. Scale bars, $20 \mu \mathrm{m}$. 


\section{Infection is cured by antibiotics treatment}

After confirming that $B$. anthracis establishes infection in silkworm and kills them, we evaluated the therapeutic effectiveness of clinically used antibiotics against $B$. anthracis infection. At first, we determined the in vitro antimicrobial susceptibility of $B$. anthracis toward a range of antibiotics. Consistent with reported studies ${ }^{42-44}$, we found that it was susceptible to most of the antibiotics tested and resistant to bacitracin and fosfomycin (Table 2).

Table 2: Antimicrobial susceptibility of Bacillus anthracis. MIC: minimum inhibitory concentration. Representative data of three independent experiments are shown.

\begin{tabular}{lc}
\hline Antimicrobial agent & MIC $(\boldsymbol{\mu g} / \mathbf{m l})$ \\
\hline Ampicillin & 0.03 \\
Bacitracin & 64 \\
Cefotaxime & 16 \\
Chloramphenicol & 4 \\
Ciprofloxacin & 0.03 \\
Doxycycline & 0.015 \\
Erythromycin & 0.5 \\
Fosfomycin & 128 \\
Gentamicin & 0.06 \\
Kanamycin & 0.5 \\
Lincomycin & 0.25 \\
Meropenem & 0.0005 \\
Rifampicin & 0.03 \\
Spectinomycin & 32 \\
Streptomycin & 0.5 \\
Tetracycline & 0.06 \\
Vancomycin & 0.008 \\
\hline
\end{tabular}

Next, we selected two antibiotics that are commonly used for the treatment of anthrax, doxycycline and ampicillin, and injected them into the hemolymph of silkworms infected with $B$. anthracis. We found that both antibiotics cured silkworms and prevented their death (Fig. 3a). We further determined the dose-response of doxycycline and ampicillin and calculated the effective doses that cure $50 \%$ of the worms $\left(E D_{50}\right) 16 \mathrm{~h}$ post-infection to be $0.05 \mathrm{mg} / \mathrm{kg}$ and $0.02 \mathrm{mg} / \mathrm{kg}$, respectively (Fig. 3b, c). We, next, determined the bacterial burden in the silkworm hemolymph at different intervals of time post-infection and found that the number of viable cells decreased with time in the antibiotic-treated groups (Fig. 3d). In addition, we checked the fluorescence expression after administering ampicillin to the BYF10124 infected larvae. We found that after 3h post- 
infection, most of the hemocytes were colonized with bacteria (Fig. 4a). While upon ampicillin treatment, only a few hemocytes were colonized with bacteria, and the overall presence of bacteria was decreased (Fig. 4b). At 6h post-infection, bacteria started proliferating outside the hemocytes (Fig. 4c), while the ampicillin treated group had fewer bacteria engulfed in the hemocytes with no bacterial growth outside the hemocytes (Fig. 4d).
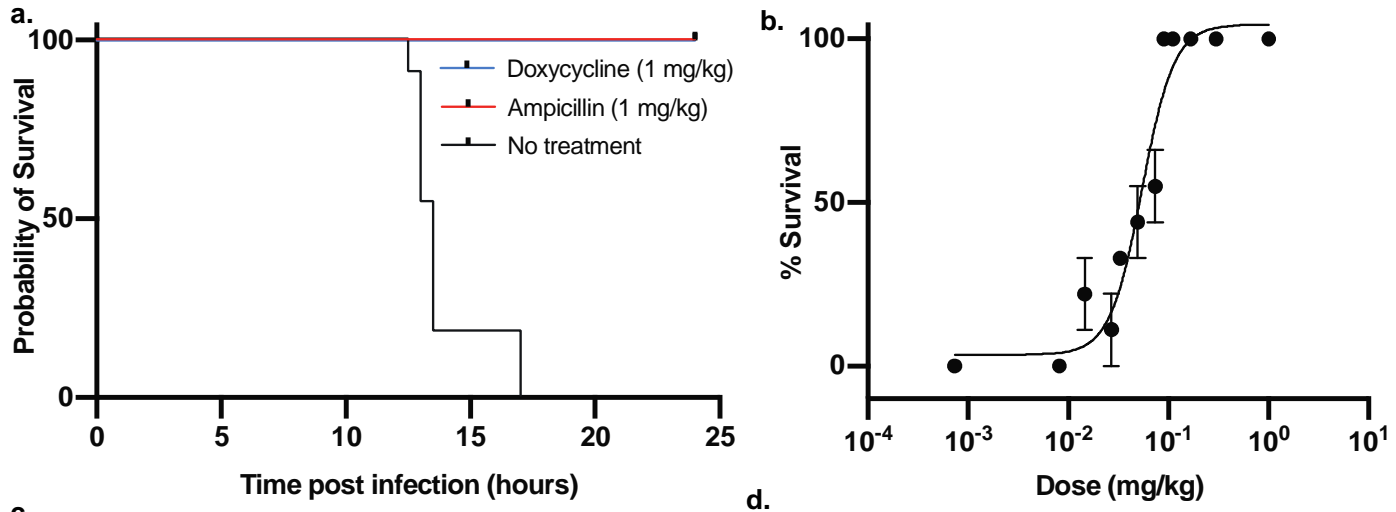

c.

d.
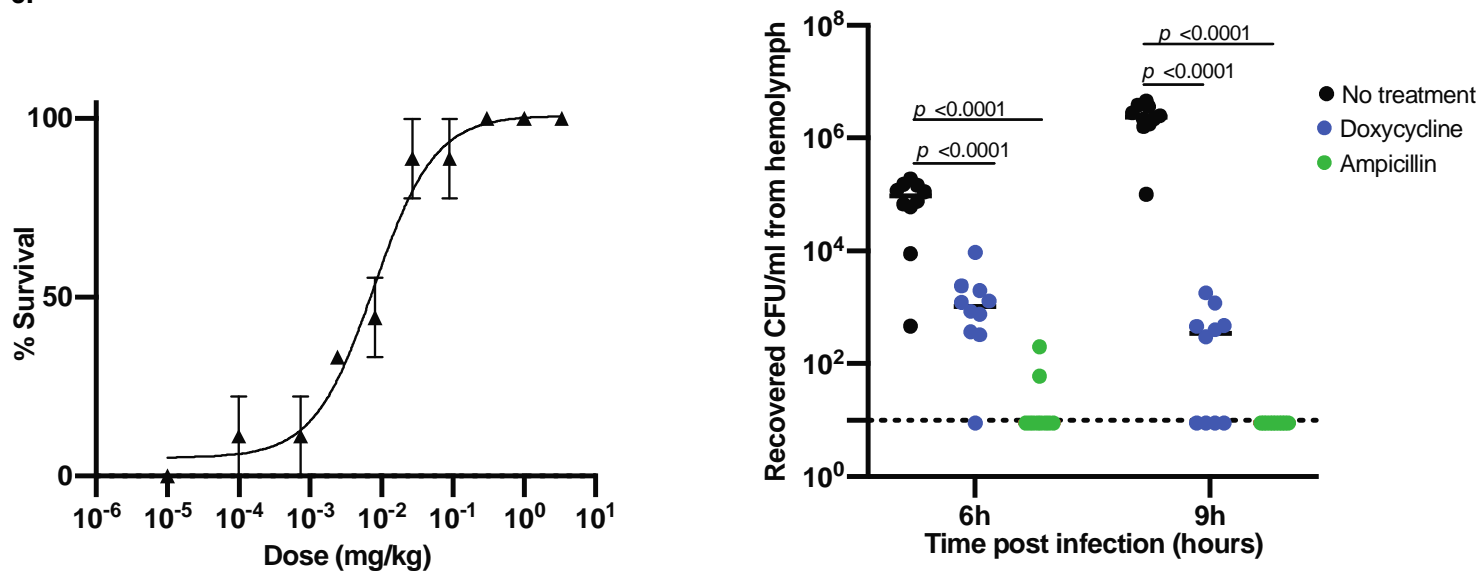

Figure 3: Treatment of $\boldsymbol{B}$. anthracis infection by antibiotics. a. Survival of silkworms $(n=10)$ with and without antibiotics treatment. Representative data from three independent experiments are shown. $\mathbf{b}, \mathbf{c}$. Survival of silkworms treated with various concentrations of doxycycline (b) or ampicillin (c) 16h postinfection. Data are mean \pm SEM of three independent experiments. $\mathrm{ED}_{50}$ value was calculated by logistic regression analysis using the logit link function. d. Bacterial burden after $6 \mathrm{~h}$ and $9 \mathrm{~h}$ post-infection with and without antibiotics treatment $(1 \mathrm{mg} / \mathrm{kg})$. Statistical analysis was performed by one-way analysis of variance (ANOVA) with Dunnett's multiple comparison test compared with the wild-type. Dotted line shows limit of detection. 
a.

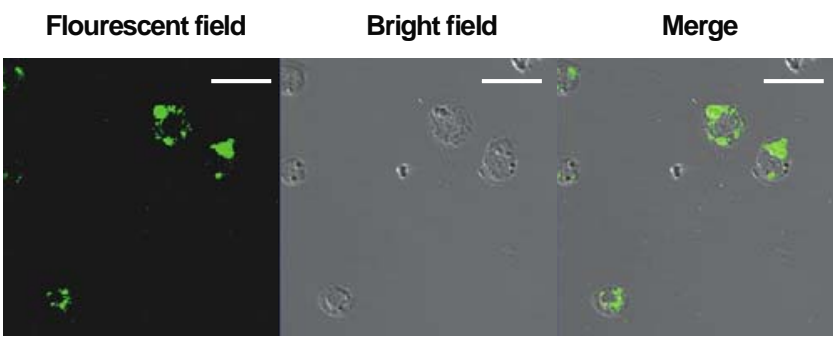

b.

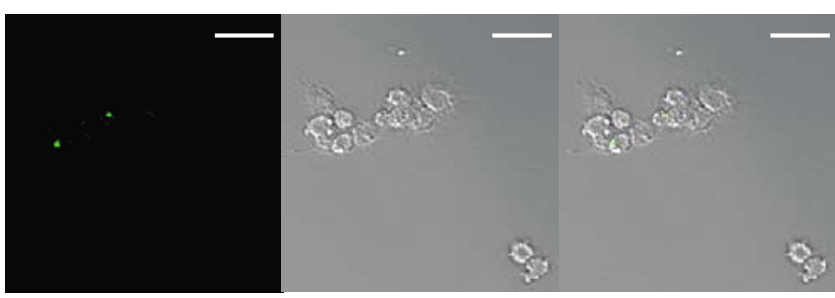

c.

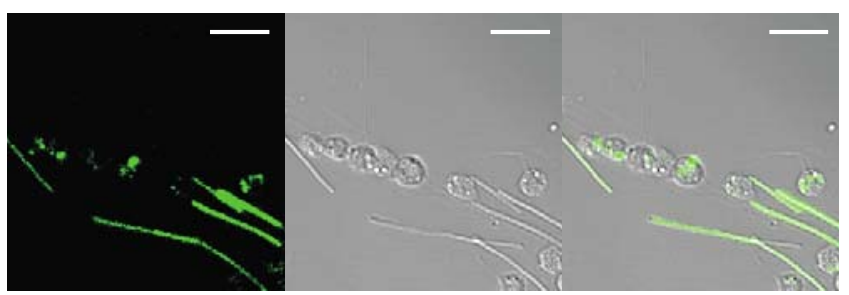

d.

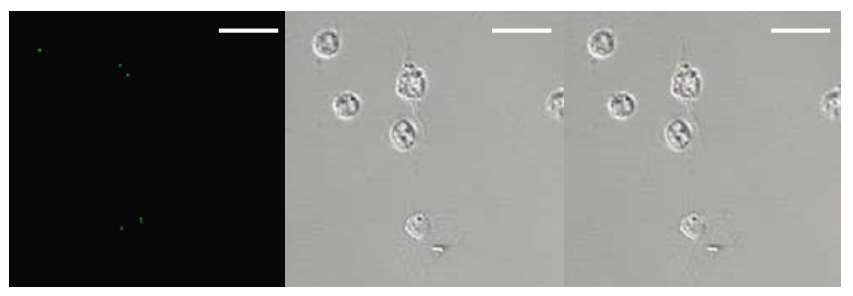

Figure 4: Clearance of $B$. anthracis from silkworm hemolymph upon antibiotic treatment. Time course of $B$. anthracis clearance by antibiotic treatment at $3 \mathrm{~h}(\mathbf{a}, \mathbf{b})$ and $6 \mathrm{~h}(\mathbf{c}, \mathbf{d})$ post-infection is shown. Silkworms were infected with BYF10124 and injected with either vehicle (a, c) or $1 \mathrm{mg} / \mathrm{kg}$ ampicillin (b, d). At specified time, hemolymph was obtained and visualized under the microscope. Scale bars, $20 \mu \mathrm{m}$.

\section{Silkworm as a model to assess virulence of $B$. anthracis}

With the establishment of the silkworm infection model of $B$. anthracis as shown above, we next used silkworms to evaluate the virulence of $B$. anthracis mutants. The toxinrelated genes are known to have roles in $B$. anthracis virulence ${ }^{45,46}$. To test whether these toxins also act on silkworms, we used mutants with disruptions in pagA, lef, and atx $A^{41}$. Located within a pathogenicity island on $\mathrm{pXO} 1^{11}$, the $p a g A$, lef, and atxA genes code for the protective antigen, the lethal factor, and a global virulence regulator AtxA, respectively. AtxA is reported to, directly and indirectly, regulate the transcription of several genes, including the $p a g A$ and lef genes ${ }^{41,47}$. We found that these mutants were 
less virulent in silkworms as they took a longer time to kill the larvae (Fig. 5a) and had attenuated colonizing ability (Fig. 5b). Taken together, it was evident that the disruption of virulence-related genes decreases the virulence of $B$. anthracis to the silkworm.

a.

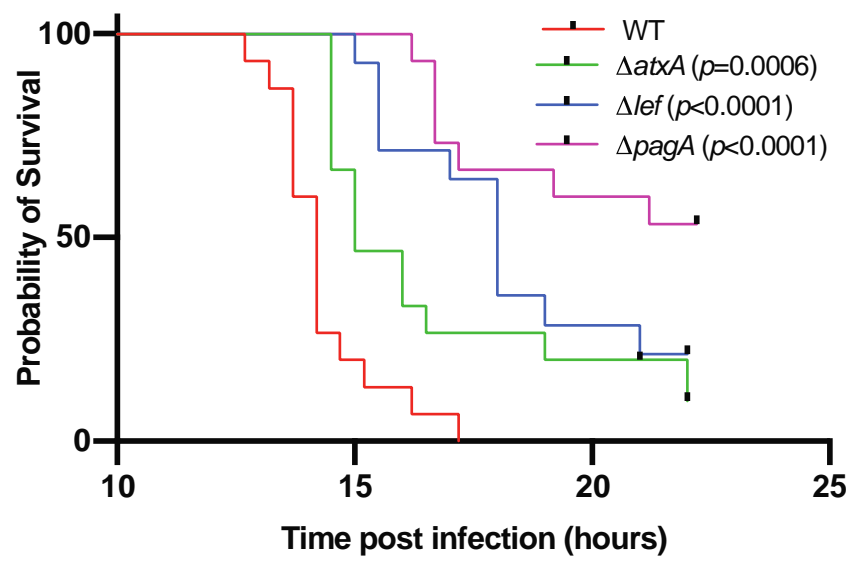

b.

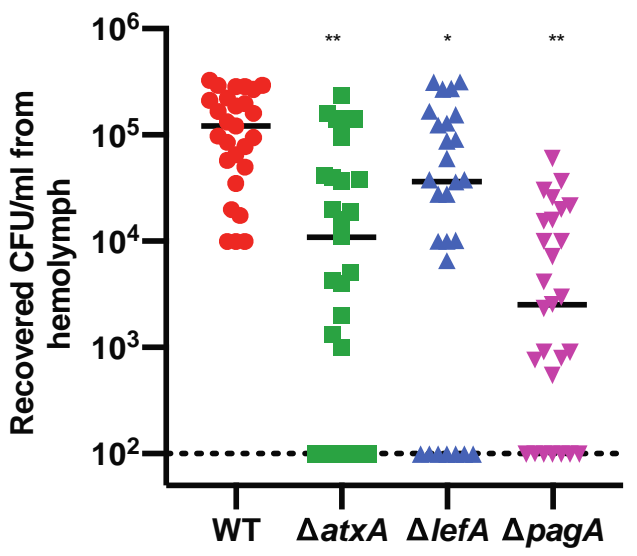

Figure 5: Assessment of virulence of $B$. anthracis mutants using silkworms. a. Survival of silkworms after infection with wild-type and virulence gene-disrupted mutants. Exponentially growing bacteria were injected into the hemolymph of silkworms and survival was observed. Data are shown as combined result from two independent experiments $(n=15)$. Injected CFU/larva: WT (wild-type) $=5 \times 10^{2}$ and $2.6 \times 10^{3}$; $\Delta$ at $x A=4 \times 10^{2}$ and $3.4 \times 10^{3} ; \Delta l e f=7.5 \times 10^{2}$ and $2 \times 10^{3} ; \Delta p a g A=4.4 \times 10^{3}$ and $1.0 \times 10^{4}$. Statistical analysis was performed by Mantel-Cox log-rank test. $\mathbf{b}$. Microbial burden of $B$. anthracis wild-type and mutants in silkworms $6 \mathrm{~h}$ post-infection. Exponentially growing bacteria were injected into the hemolymph of silkworms, hemolymph was recovered 6h post-infection and CFU was determined. Data are shown as combined result from two independent experiments $(\mathrm{n}=27)$. Injected CFU/larva: WT (wild-type) $=2 \times 10^{2}$ and $3 \times 10^{2} ; \Delta$ atx $A=1.8 \times 10^{2}$ and $4.4 \times 10^{2} ; \Delta l e f=2.2 \times 10^{2}$ and $2.4 \times 10^{2} ; \Delta$ pag $A=1.1 \times 10^{2}$ and $2.8 \times$ $10^{2}$. Statistical analysis was performed by one-way analysis of variance (ANOVA) with Dunnett's multiple comparison test compared with the WT $\left({ }^{*} p<0.05,{ }^{* *} p<0.0001\right)$. Dotted line represents limit of detection.

\section{Discussion}

In this study, we established a silkworm model of $B$. anthracis infection and evaluated the therapeutic effects of clinically used antibiotics in silkworms infected with $B$. anthracis. Moreover, we generated a $B$. anthracis strain expressing AmCyan1, which was useful in evaluating the proliferation of bacteria inside the host over time. While silkworm infection models of human pathogens have been reported ${ }^{30,32,48-50}$, this is the first report of the silkworm infection model of $B$. anthracis. We found that $B$. anthracis Sterne killed silkworms and the $\mathrm{LD}_{50}$ was $8.3 \times 10^{2} \mathrm{CFU}$, which was comparable with those in mice models where $\mathrm{LD}_{50}$ ranged from $1.6 \times 10^{2}-1.1 \times 10^{3} \mathrm{CFU}^{51,52}$. Live 
bacteria were required for silkworm killing, which was evident from the fact that heatkilled bacteria $\left(10^{6} \mathrm{CFU}\right)$ could not kill the larvae. B. anthracis established infection within silkworms as their proliferation was increased over time observed both from the increased CFU in the hemolymph of larvae and the increased proliferation of fluorescent bacteria under the microscope harvested at various intervals post-infection.

When we administered clinically used antibiotics to the $B$. anthracis infected silkworms in this study, we observed therapeutic activities of the antibiotics as they prolonged the survival of infected silkworms. Recovered CFU of bacteria from the treated silkworm hemolymph showed a faster clearance of bacteria in the ampicillin-treated group than in the doxycycline-treated group. As ampicillin is a bactericidal antibiotic, bacteria are killed in addition to clearance from the host immunity, which may have led to faster overall clearance; whereas, being a bacteriostatic antibiotic, doxycycline inhibited the bacteria growth, and overall clearance may have depended upon the host immunity taking a longer time. We further demonstrated, using fluorescent protein-expressing $B$. anthracis, that antibiotic treatment reduces bacterial burden in the hemolymph. The therapeutic effects of known antibiotics in the silkworm infection model imply that the therapeutic effectiveness of unknown compounds can be evaluated using this system, selecting for compounds with therapeutic activity and appropriate pharmacokinetics at an early stage of screening ${ }^{35,36,38}$. An additional advantage of using silkworms is that a small quantity of compounds would be enough to evaluate therapeutic effectiveness.

The innate immunity is partly conserved among silkworms and mammals, and several signaling cascades such as the mitogen-activated protein kinase (MAPK) pathways are activated in silkworms by bacterial components resulting in antimicrobial peptides production $^{53}$. Thus, silkworms could differentiate the virulence of the mutant deficient in the lethal factor of $B$. anthracis, which acts via the MAPK pathway ${ }^{54}$. However, silkworms do not have acquired immunity, and therefore, they can be used to determine virulence factors that trigger the innate immunity and not the acquired immunity. Nonetheless, innate immunity is the first line of defense in all organisms ${ }^{55}$, and evaluation of virulence factors of pathogenic microorganisms have been performed 
using silkworm model ${ }^{28,30,56-58}$. The finding of this study showing attenuated virulence of strains of $B$. anthracis with disruption in known virulence genes suggested that silkworms can be used to evaluate the roles of unknown genes in the virulence of $B$. anthracis.

Since Sterne strain lacks pXO2 and is less virulent to higher animals ${ }^{40}$, silkworm model of $B$. anthracis Sterne infection will have an additional advantage for the identification of virulence factors encoded by genes either in the chromosome or pXO1 that might be masked in a highly virulent strain containing both $\mathrm{pXO} 1$ and $\mathrm{pXO}$. The identified virulence factors can then be utilized as potential vaccine targets in Sterne strain such as $h t r A$, aro, nos, sod, and $c / p X^{14,59-62}$. As the use of Sterne strain in humans is associated with safety concerns, the identification of other virulence factors may contribute to the development of a safe human vaccine strain.

\section{Acknowledgments}

We thank Dr. Takeshi Saito (International Institute for Zoonosis Control, Hokkaido University, Japan) for help with fluorescent microscopy and Tomoko Shimizu (International Institute for Zoonosis Control, Hokkaido University, Japan) for technical assistance.

\section{Funding}

This work was supported by JSPS KAKENHI Grant Number JP19K16653 and JP21K15430 to AP, JP18K14672 to YF, and JP18K19436 to HH and AMED Grant number JP21wm0125008 (the Japan Program for Infectious Diseases Research and Infrastructure) to $\mathrm{AP}, \mathrm{YF}$, and $\mathrm{HH}$.

\section{Declaration of interest statement}

The authors declare no conflict of interest. 


\section{References:}

1 Fritze, D. Taxonomy of the genus bacillus and related genera: the aerobic endosporeforming bacteria. Phytopathology 94, 1245-1248, doi:10.1094/phyto.2004.94.11.1245 (2004).

2 Muturi, M. et al. Recurrent Anthrax Outbreaks in Humans, Livestock, and Wildlife in the Same Locality, Kenya, 2014-2017. The American journal of tropical medicine and hygiene 99, 833-839, doi:10.4269/ajtmh.18-0224 (2018).

$3 \mathrm{Yu}, \mathrm{D}$. et al. Investigation and source-tracing of an anthrax outbreak in Gansu Province, China. PLoS One 13, e0203267, doi:10.1371/journal.pone.0203267 (2018).

$4 \quad$ Lewerin, S. S. et al. Anthrax outbreak in a Swedish beef cattle herd--1st case in 27 years: Case report. Acta veterinaria Scandinavica 52, 7, doi:10.1186/1751-0147-52-7 (2010).

5 Hang'ombe, M. B. et al. Human-animal anthrax outbreak in the Luangwa valley of Zambia in 2011. Tropical doctor 42, 136-139, doi:10.1258/td.2012.110454 (2012).

$6 \quad$ Nayak, P. et al. A cutaneous Anthrax outbreak in Koraput District of Odisha-India 2015. BMC public health 19, 470, doi:10.1186/s12889-019-6787-0 (2019).

7 Thapa, N. K. et al. Investigation and control of anthrax outbreak at the human-animal interface, Bhutan, 2010. Emerging Infectious Diseases 20, 1524-1526, doi:10.3201/eid2009.140181 (2014).

8 Hanczaruk, M. et al. Injectional anthrax in heroin users, Europe, 2000-2012. Emerging Infectious Diseases 20, 322-323, doi:10.3201/eid2002.120921 (2014).

9 Navdarashvili, A. et al. Human anthrax outbreak associated with livestock exposure: Georgia, 2012. Epidemiology and infection 144, 76-87, doi:10.1017/s0950268815001442 (2016).

10 Jernigan, D. B. et al. Investigation of bioterrorism-related anthrax, United States, 2001: epidemiologic findings. Emerging Infectious Diseases 8, 1019-1028, doi:10.3201/eid0810.020353 (2002).

11 Okinaka, R. T. et al. Sequence and organization of pX01, the large Bacillus anthracis plasmid harboring the anthrax toxin genes. J Bacteriol 181, 6509-6515, doi:10.1128/jb.181.20.6509-6515.1999 (1999).

12 Makino, S., Uchida, I., Terakado, N., Sasakawa, C. \& Yoshikawa, M. Molecular characterization and protein analysis of the cap region, which is essential for encapsulation in Bacillus anthracis. J Bacteriol 171, 722-730, doi:10.1128/jb.171.2.722730.1989 (1989).

13 Gat, O. et al. The solute-binding component of a putative Mn(II) ABC transporter (MntA) is a novel Bacillus anthracis virulence determinant. Mol Microbiol 58, 533-551, doi:10.1111/j.1365-2958.2005.04848.x (2005).

14 McGillivray, S. M. et al. ClpX contributes to innate defense peptide resistance and virulence phenotypes of Bacillus anthracis. Journal of innate immunity 1, 494-506, doi:10.1159/000225955 (2009).

15 Kern, J. \& Schneewind, O. BsIA, the S-layer adhesin of B. anthracis, is a virulence factor for anthrax pathogenesis. Mol Microbiol 75, 324-332, doi:10.1111/j.13652958.2009.06958.x (2010). 
16 Chitlaru, T. et al. HtrA is a major virulence determinant of Bacillus anthracis. Mol Microbiol 81, 1542-1559, doi:10.1111/j.1365-2958.2011.07790.x (2011).

17 Jenkins, A. et al. Role of purine biosynthesis in Bacillus anthracis pathogenesis and virulence. Infect Immun 79, 153-166, doi:10.1128/iai.00925-10 (2011).

18 Israeli, M. et al. Distinct Contribution of the HtrA Protease and PDZ Domains to Its Function in Stress Resilience and Virulence of Bacillus anthracis. Front Microbiol 10, 255, doi:10.3389/fmicb.2019.00255 (2019).

19 Begun, J., Sifri, C. D., Goldman, S., Calderwood, S. B. \& Ausubel, F. M. Staphylococcus aureus virulence factors identified by using a high-throughput Caenorhabditis eleganskilling model. Infect Immun 73, 872-877, doi:10.1128/IAI.73.2.872-877.2005 (2005).

20 Diard, M. et al. Caenorhabditis elegans as a simple model to study phenotypic and genetic virulence determinants of extraintestinal pathogenic Escherichia coli. Microbes and infection 9, 214-223, doi:10.1016/j.micinf.2006.11.009 (2007).

21 Tan, M. W., Rahme, L. G., Sternberg, J. A., Tompkins, R. G. \& Ausubel, F. M. Pseudomonas aeruginosa killing of Caenorhabditis elegans used to identify $P$. aeruginosa virulence factors. Proc Natl Acad Sci U S A 96, 2408-2413, doi:10.1073/pnas.96.5.2408 (1999).

22 Younes, S., Al-Sulaiti, A., Nasser, E. A. A., Najjar, H. \& Kamareddine, L. Drosophila as a Model Organism in Host-Pathogen Interaction Studies. Front Cell Infect Microbiol 10, 214, doi:10.3389/fcimb.2020.00214 (2020).

23 Lee, Y. J., Jang, H. J., Chung, I. Y. \& Cho, Y. H. Drosophila melanogaster as a polymicrobial infection model for Pseudomonas aeruginosa and Staphylococcus aureus. Journal of microbiology (Seoul, Korea) 56, 534-541, doi:10.1007/s12275-018-8331-9 (2018).

24 Needham, A. J., Kibart, M., Crossley, H., Ingham, P. W. \& Foster, S. J. Drosophila melanogaster as a model host for Staphylococcus aureus infection. Microbiology (Reading) 150, 2347-2355, doi:10.1099/mic.0.27116-0 (2004).

25 Malmquist, J. A., Rogan, M. R. \& McGillivray, S. M. Galleria mellonella as an Infection Model for Bacillus anthracis Sterne. Front Cell Infect Microbiol 9, 360, doi:10.3389/fcimb.2019.00360 (2019).

26 Cools, F. et al. Optimization and Characterization of a Galleria mellonella Larval Infection Model for Virulence Studies and the Evaluation of Therapeutics Against Streptococcus pneumoniae. Front Microbiol 10, 311, doi:10.3389/fmicb.2019.00311 (2019).

27 Sheehan, G., Dixon, A. \& Kavanagh, K. Utilization of Galleria mellonella larvae to characterize the development of Staphylococcus aureus infection. Microbiology (Reading) 165, 863-875, doi:10.1099/mic.0.000813 (2019).

28 Paudel, A., Hamamoto, H., Panthee, S., Matsumoto, Y. \& Sekimizu, K. Large-scale screening and identification of novel pathogenic Staphylococcus aureus genes using a silkworm infection model. J. Infect. Dis. 221, 1795-1804 (2020).

29 Paudel, A., Panthee, S., Hamamoto, H., Grunert, T. \& Sekimizu, K. YjbH regulates virulence genes expression and oxidative stress resistance in Staphylococcus aureus. Virulence 12, 470-480, doi:10.1080/21505594.2021.1875683 (2021).

30 Kaito, C. et al. Silkworm pathogenic bacteria infection model for identification of novel virulence genes. Mol. Microbiol. 56, 934-944, doi:10.1111/j.1365-2958.2005.04596.x (2005). 
31 Panthee, S., Paudel, A., Hamamoto, H. \& Sekimizu, K. Advantages of the silkworm as an animal model for developing novel antimicrobial agents. Front. Microbiol. 8, 373 (2017).

32 Hamamoto, H. et al. Quantitative evaluation of the therapeutic effects of antibiotics using silkworms infected with human pathogenic microorganisms. Antimicrob. Agents Chemother. 48, 774-779, doi:10.1128/aac.48.3.774-779.2004 (2004).

33 Matsumoto, Y., Ishii, M., Hasegawa, S. \& Sekimizu, K. Enterococcus faecalis YM0831 suppresses sucrose-induced hyperglycemia in a silkworm model and in humans. Commun Biol 2, 157, doi:10.1038/s42003-019-0407-5 (2019).

34 Zhang, X. et al. Silkworms can be used as an animal model to screen and evaluate gouty therapeutic drugs. J. Insect. Sci. 12 (2012).

35 Hamamoto, $H$. et al. Lysocin $E$ is a new antibiotic that targets menaquinone in the bacterial membrane. Nat. Chem. Biol. 11, 127-133 (2015).

36 Paudel, A. et al. A novel spiro-heterocyclic compound identified by the silkworm infection model inhibits transcription in Staphylococcus aureus. Front. Microbiol. 8, 712, doi:10.3389/fmicb.2017.00712 (2017).

37 Paudel, A., Panthee, S., Hamamoto, H. \& Sekimizu, K. GPI0363 inhibits the interaction of RNA polymerase with DNA in Staphylococcus aureus. RSC Adv. 9, 37889-37894, doi:10.1039/C9RA06844A (2019).

38 Paudel, A. et al. Pharmacokinetic parameters explain the therapeutic activity of antimicrobial agents in a silkworm infection model. Sci. Rep. 8, 1578, doi:10.1038/s41598-018-19867-0 (2018).

39 Plaut, R. D. \& Stibitz, S. Improvements to a Markerless Allelic Exchange System for Bacillus anthracis. PLoS One 10, e0142758, doi:10.1371/journal.pone.0142758 (2015).

40 Sterne, M. Avirulent anthrax vaccine. The Onderstepoort journal of veterinary science and animal industry 21, 41-43 (1946).

41 Yoshikazu Furuta, C. C., Tuvshinzaya Zorigt, Atmika Paudel, Shun Izumi, Mai Tsujinouchi, Tomoko Shimizu, Wim Meijer, Hideaki Higashi. Direct regulons of AtxA, the master virulence regulator of Bacillus anthracis. Submitted (2021).

42 Frean, J., Klugman, K. P., Arntzen, L. \& Bukofzer, S. Susceptibility of Bacillus anthracis to eleven antimicrobial agents including novel fluoroquinolones and a ketolide. J Antimicrob Chemother 52, 297-299, doi:10.1093/jac/dkg364 (2003).

43 Furuta, Y. et al. Loss of Bacitracin Resistance Due to a Large Genomic Deletion among Bacillus anthracis Strains. mSystems 3, doi:10.1128/mSystems.00182-18 (2018).

44 Athamna, A. et al. In vitro susceptibility of Bacillus anthracis to various antibacterial agents and their time-kill activity. J Antimicrob Chemother 53, 247-251, doi:10.1093/jac/dkh016 (2004).

45 Levy, H. et al. Differential contribution of Bacillus anthracis toxins to pathogenicity in two animal models. Infect Immun 80, 2623-2631, doi:10.1128/iai.00244-12 (2012).

46 Levy, H. et al. Toxin-independent virulence of Bacillus anthracis in rabbits. PLoS One 9, e84947, doi:10.1371/journal.pone.0084947 (2014).

47 Koehler, T. M. Bacillus anthracis genetics and virulence gene regulation. Curr Top Microbiol Immunol 271, 143-164, doi:10.1007/978-3-662-05767-4_7 (2002). 
48 Hosoda, K. et al. Evaluation of Anti-Mycobacterial Compounds in a Silkworm Infection Model with Mycobacteroides abscessus. Molecules 25, doi:10.3390/molecules25214971 (2020).

49 Matsumoto, Y. \& Sekimizu, K. Silkworm as an experimental animal for research on fungal infections. Microbiol Immunol 63, 41-50, doi:10.1111/1348-0421.12668 (2019).

50 Yagi, A. et al. Development of an in vivo-mimic silkworm infection model with Mycobacterium avium complex. Drug Discov Ther 14, 287-295, doi:10.5582/ddt.2020.03099 (2021).

51 Welkos, S. L., Keener, T. J. \& Gibbs, P. H. Differences in susceptibility of inbred mice to Bacillus anthracis. Infect Immun 51, 795-800, doi:10.1128/iai.51.3.795-800.1986 (1986).

52 Chitlaru, T. et al. Next-Generation Bacillus anthracis Live Attenuated Spore Vaccine Based on the htrA(-) (High Temperature Requirement A) Sterne Strain. Sci Rep 6, 18908, doi:10.1038/srep18908 (2016).

53 Ishii, K. et al. Insect cytokine paralytic peptide (PP) induces cellular and humoral immune responses in the silkworm Bombyx mori. J Biol Chem 285, 28635-28642, doi:10.1074/jbc.M110.138446 (2010).

54 Tonello, F. \& Montecucco, C. The anthrax lethal factor and its MAPK kinase-specific metalloprotease activity. Mol Aspects Med 30, 431-438, doi:10.1016/j.mam.2009.07.006 (2009).

55 Chaplin, D. D. Overview of the immune response. The Journal of allergy and clinical immunology 125, S3-23, doi:10.1016/j.jaci.2009.12.980 (2010).

56 Hanaoka, N. et al. Identification of the putative protein phosphatase gene PTC1 as a virulence-related gene using a silkworm model of Candida albicans infection. Eukaryotic cell 7, 1640-1648, doi:10.1128/ec.00129-08 (2008).

57 Chieda, Y. et al. Pathogenicity of gacA mutant of Pseudomonas aeruginosa PA01 in the silkworm, Bombyx mori. FEMS Microbiol Lett 244, 181-186, doi:10.1016/j.femsle.2005.01.032 (2005).

58 Tran, S. L. et al. Haemolysin II is a Bacillus cereus virulence factor that induces apoptosis of macrophages. Cellular microbiology 13, 92-108, doi:10.1111/j.14625822.2010.01522.x (2011).

59 Chitlaru, T. et al. A novel live attenuated anthrax spore vaccine based on an acapsular Bacillus anthracis Sterne strain with mutations in the htrA, lef and cya genes. Vaccine 35, 6030-6040, doi:10.1016/j.vaccine.2017.03.033 (2017).

60 Ivins, B. E., Welkos, S. L., Knudson, G. B. \& Little, S. F. Immunization against anthrax with aromatic compound-dependent (Aro-) mutants of Bacillus anthracis and with recombinant strains of Bacillus subtilis that produce anthrax protective antigen. Infect Immun 58, 303-308, doi:10.1128/iai.58.2.303-308.1990 (1990).

61 Shatalin, K. et al. Bacillus anthracis-derived nitric oxide is essential for pathogen virulence and survival in macrophages. Proc Natl Acad Sci U S A 105, 1009-1013, doi:10.1073/pnas.0710950105 (2008).

62 Cybulski, R. J., Jr. et al. Four superoxide dismutases contribute to Bacillus anthracis virulence and provide spores with redundant protection from oxidative stress. Infect Immun 77, 274-285, doi:10.1128/iai.00515-08 (2009). 